\title{
Bridging length-scales from molecules to tissues using mouse genetics, cryoCLEM, and cryoET
}

Julia Peukes ${ }^{1}$, Megan Lovatt ${ }^{2}$, Conny Leistner ${ }^{2}$, Dustin Morado ${ }^{3}$, Fei Zhu ${ }^{4}$, Jerome Boulanger ${ }^{5}$, Wanda Kukulski $^{6}$, Noboru Komiyama ${ }^{4}$, Seth Grant ${ }^{4}$, John Briggs ${ }^{5}$ and Rene Frank ${ }^{7}$

${ }^{1}$ MRC Laboratory of Molecular Biology, Cambridge, United Kingdom, ${ }^{2}$ University of Leeds, United Kingdom, ${ }^{3}$ SciLifeLab, Sweden, ${ }^{4}$ University of Edinburgh, United Kingdom, ${ }^{5} \mathrm{MRC}$ Laboratory of Molecular Biology, United Kingdom, ${ }^{6}$ University of Bern, Switzerland, ${ }^{7}$ University of Leeds, Leeds, United Kingdom

Synapses are specialized, submicron-sized cell-cell contacts that wire-up neuronal circuits and mediate the storage of information. Most synaptic communication is via chemical neurotransmitters, of which glutamate is the major excitatory neurotransmitter in the mammalian brain. Glutamate is packaged and released from the presynaptic terminal and detected by glutamate receptors in the postsynaptic membrane. The functional output of synapses is determined directly by the molecular composition and structure of the postsynaptic compartment, which is thought to be responsible for the acquisition of memory.

For over half a century conventional electron microscopy of chemically fixed, dehydrated, and heavy metal-stained tissues has led to the ultrastructural identification of glutamatergic synapses. Glutamatergic synapses in these samples are characterized by an extraordinary concentration of proteins associated with postsynaptic compartment, called the postsynaptic density (Sheng \& Kim, 2011; Gray, 1959).

To obtain structural insights into the native 3D molecular architecture of fresh glutamatergic synapses in the adult mouse brain we developed workflows combining mouse genetics, cryogenic correlated light and electron microscopy (cryoCLEM) and cryo-electron tomography.

To locate mature glutamatergic synapses within the brain we generated a $P s d 95^{E G F P}$ knockin mouse. PSD95 is a scaffold protein that anchors glutamate receptors at the postsynaptic membrane. The $P s d 95^{E G F P}$ mouse line expresses endogenous PSD95 engineered with an in-frame C-terminal GFP fusion, which marks the postsynaptic membrane without over-expression. Crucially, this mutant mouse line was examined in biochemical, anatomical, and electrophysiological assays, which indicated that while leaving the anatomical expression and synaptic functions unperturbed, GFP-tagged PSD95 marked the location of glutamatergic synapses (Zhu et al., 2018; Broadhead et al., 2016; Frank et al., 2016).

To prepare synapses rapidly $(<2 \mathrm{~min}$ ) for cryoEM, brain tissue samples were homogenized in ice-cold artificial cerebrospinal fluid (ACSF), blotted, and vitrified on cryoEM grids. Synapses within this complex mixture were located on the cryoEM grid by cryogenic fluorescent light microscopy (cryoFM, Fig. 1A,B). Next, fluorescent locations were mapped in the electron microscope by cryoCLEM. This mapping procedure has a precision of $\sim 300 \mathrm{~nm}$, much smaller than the size of an individual synapse (Kukulski et al., 2011). Finally, fluorescent loci were used to direct the collection of cryo-electron tomograms. The reconstructed 3D volumes from these data revealed in exquisite detail the macromolecular architecture of the adult brain synapses (Fig. 1C,D), including organelles, individual glutamate receptors, cytoskeletal elements, and many other multiprotein complexes (Fig. 1C,D).

To investigate the native molecular architecture of synapses in anatomically intact brain, tissue biopsies of adult $P s d 95^{E G F P}$ cortex were high-pressure frozen, from which thin, vitreous cryo-sections were 
collected (Zuber et al., 2005). Using our cryoCLEM and cryoET workflow, we determined the in-tissue 3D architecture of glutamatergic synapses in the mammalian brain.

Overall, these complementary high-fidelity workflows enable the elucidation of molecular architectures of genetically-defined constituents within the mammalian brain.

A

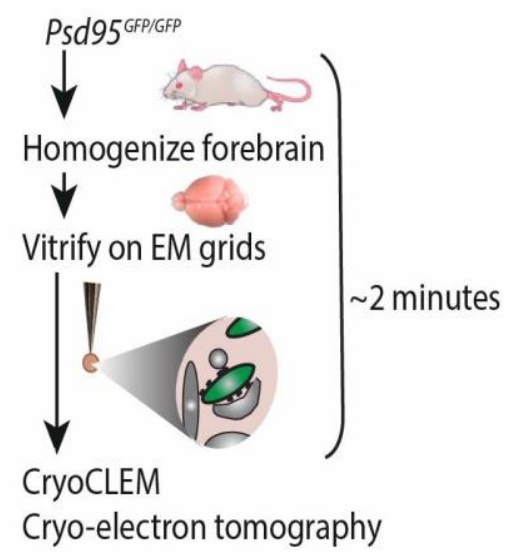

C

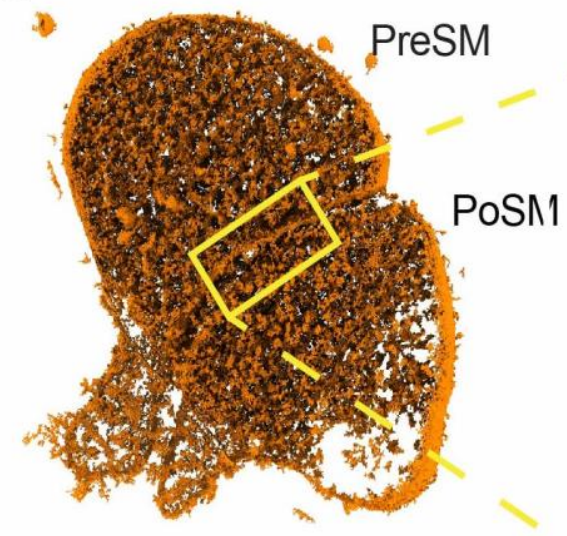

B



D

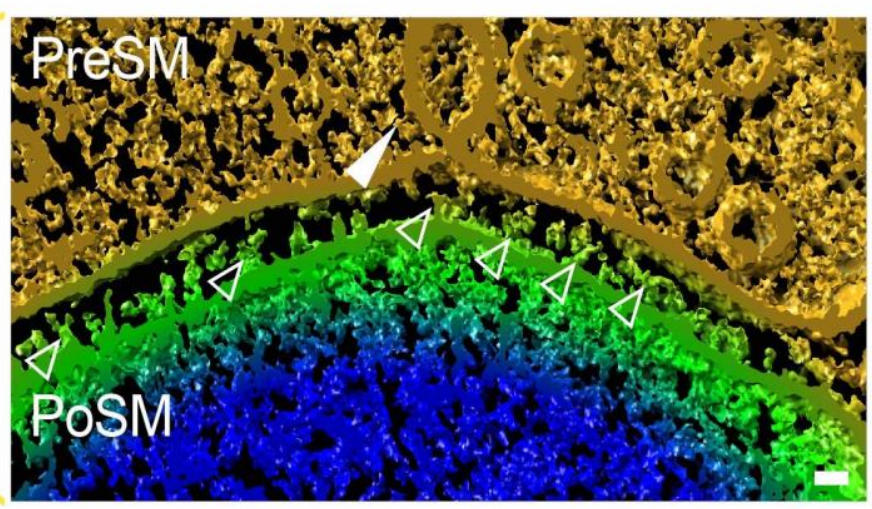

$10 \mathrm{~nm}$

Figure 1. Molecular structure of mouse brain synapses. A) Schematic showing preparaton of PSD95-GFP labelled synapses for cryogenic correlated light-electron microscopy (cryoCLEM) and cryogenic electron tomography (cryoET) with Volta phase-plate. B) CryoCLEM to locate PSD95-GFP, which marks postsynaptic membrane (PoSM) that apposes presynaptic membrane (PreSM). C) Raw 3D tomographic map of synapse with D), close up virtual slice through the tomographic map. Yellow, presynaptic membrane, including synaptic vesicles. Green, postsynaptic membrane. Blue, luminal postsynaptic cytoplasm. Maps shows proteins, including putative glutamate receptors (open arrow heads), cytoskeleton and numerous other macromolecular complexes. Arrow head, intermediate of membrane fission/fusion.

References

Broadhead, M. J., Horrocks, M. H., Zhu, F., Muresan, L., Benavides-Piccione, R., DeFeliPe, J., Fricker, D., Kopanitsa, M. V., Duncan, R. R., Klenerman, D., Komiyama, N. H., LeE, S. F. \& Grant, 
S. G. N. (2016). PSD95 nanoclusters are postsynaptic building blocks in hippocampus circuits. Scientific Reports 6, 1-14.

Frank, R. A. W., Komiyama, N. H., Ryan, T. J., Zhu, F., Dell, T. J. O. R. \& Grant, S. G. N. (2016). NMDA receptors are selectively partitioned into complexes and supercomplexes during synapse maturation. Nature Communications 7, 11264.

GrAY, E. G. (1959). Axo-somatic and axo-dendritic synapses of the cerebral cortex: An electron microscope study. Journal of anatomy 93, 420-433.

Kukulski, W., Schorb, M., Welsch, S., Picco, A., KaKsonen, M. \& BrigGS, J. A. G. (2011). Correlated fluorescence and 3D electron microscopy with high sensitivity and spatial precision. The Journal of Cell Biology 192, 111-119.

ShEnG, M. \& KIM, E. (2011). The Postsynaptic Organization of Synapses. Cold Spring Harbor Perspectives in Biology 3, 1-21.

Zhu, F., Cizeron, M., Qiu, Z., Benavides-Piccione, R., Kopanitsa, M. V., Skene, N. G., Koniaris, B., DeFelipe, J., Fransén, E., Komiyama, N. H. \& Grant, S. G. N. (2018). Architecture of the Mouse Brain Synaptome. Neuron 99, 781-799.

Zuber, B., Nikonenko, I., Klauser, P., Muller, D. \& Dubochet, J. (2005). The mammalian central nervous synaptic cleft contains a high density of periodically organized complexes. Proceedings Of The National Academy Of Sciences Of The United States Of America 102, 19192-19197. 